\title{
Pilot study of the efficacy and safety of lettuce seed oil in patients with sleep disorders
}

\author{
This article was published in the following Dove Press journal: \\ International Journal of General Medicine \\ 9 June 2011 \\ Number of times this article has been viewed
}

\author{
Mostafa Yakoot' \\ Sherine Helmy ${ }^{2}$ \\ Kamal Fawal ${ }^{3}$ \\ 'Green Clinic Research Center, \\ ${ }^{2}$ Pharco Pharmaceutical Company, \\ ${ }^{3}$ Mamorah Psychiatric Hospital, \\ Alexandria, Egypt
}

\begin{abstract}
Background: Lactuca sativa (garden lettuce) is a popular salad herb. It has been in use in folk medicine since ancient times as both an appetite stimulant and as an aid to sleep. L. sativa seed oil $\left(\operatorname{Sedan}^{\circledR}\right)$ has demonstrated a pronounced sedative effect and potentiated the hypnotic effect of barbiturates in animal models. It also exhibited significant analgesic and anti-inflammatory activities. In this study, we evaluated the sedative and hypnotic effects of $L$. sativa in patients suffering from insomnia.
\end{abstract}

Methods: Sixty patients suffering from insomnia with or without anxiety were randomized to receive capsules containing L. sativa seed oil $1000 \mathrm{mg}(\mathrm{n}=30)$ or placebo $(\mathrm{n}=30)$. All patients were asked to complete a verbal questionnaire before the start of the trial and 1 week after starting treatment.

Results: Improvements in the modified State-Trait Anxiety Inventory and the Sleep rating scale scores were significantly greater in patients receiving $L$. sativa seed oil compared with those on placebo $(P<0.05)$. No side effects were found to be attributable to $L$. sativa seed oil at the given dosage.

Conclusion: L. sativa seed oil was found to be a useful sleeping aid and may be a hazard-free line of treatment, especially in geriatric patients suffering from mild-to-moderate forms of anxiety and sleeping difficulties.

Keywords: Lactuca sativa seed oil, insomnia, sleeping disorder, anxiety

\section{Introduction}

Sleep loss and sleep disorders are among the most common health problems, but are frequently overlooked. It is estimated that about one-third of people in the general population suffer from a chronic disorder of sleep and wakefulness. ${ }^{1}$ Insomnia is a subjective complaint that sleep is difficult to initiate or maintain, or that it is nonrefreshing or of poor quality and quantity. ${ }^{2}$

Insomnia affects approximately $20 \%-40 \%$ of older adults at least a few nights per month. ${ }^{3,4}$ It is an important concern in elderly patients, not only because it is common, but also because it can cause multiple daytime impairments, such as difficulty sustaining attention or alertness, a slowed response time, impaired memory and concentration, and decreased performance. Insomnia is also associated with a shortened lifespan because of an increased risk of heart disease, stroke, cancer, and suicide. ${ }^{5,6}$

Insomnia is mostly treated with benzodiazepine and nonbenzodiazepine hypnotic drugs, which potentiate the central nervous system suppressant activity of gamma aminobutyric acid A receptors in the brain. However, residual daytime effects, development of dependence, and withdrawal symptoms associated with most hypnotic drugs are of considerable concern in public health. ${ }^{7}$
Correspondence: Mostafa Yakoot Green Clinic Research Center,

27 Green Street,

Alexandria 21121 , Egypt

Tel +20 I 2392756 I

Fax +20133913725

Emailyakoot@yahoo.com 
Lactuca sativa (garden lettuce), a member of the Compositae family, is a very popular salad herb in Egypt. ${ }^{8,9}$ Its seed oil has been in use in folk medicine since ancient times as a sleeping aid, as well as to relieve pain and inflammation. ${ }^{10,11}$

A crude methanol/petroleum ether extract from L. sativa seeds has demonstrated a time-dependent and dose-dependent analgesic effect in a formalin test and also dose-dependent anti-inflammatory activity in a carrageenan model of inflammation. ${ }^{12}$

L. sativa seed oil $\left(\operatorname{Sedan}^{\circledR}\right.$, Pharco Pharmaceutical Company, Alexandria, Egypt) is marketed as a soft gelatin capsule containing $1000 \mathrm{mg}$ of the purified expressed oil. Gas liquid chromatography shows that the L. sativa seed oil capsule is comprised mainly of oleic acid (61.5\%), stearic acid (20.4\%), palmitic acid (9.7\%), myristic acid (2.8\%), cis-palmitoleic (1.2\%), behenic acid (0.5\%), and lignoseric acid $(0.3 \%)$, with B-sitosterol and B-amryn also present.

Despite its well-known safety over thousands of years of use, $L$. sativa seed oil has only recently been subjected to extensive pharmacological and toxicological investigation. It was found to have significant sedative, analgesic, and antiinflammatory activity in a murine model, ${ }^{13}$ and in the same study was shown to have significant anticonvulsant activity against convulsions induced by pentylenetetrazole and to potentiate the hypnotic effect of barbiturates. The oral $\mathrm{LD}_{50}$ was found to be $19.75 \mathrm{~mL} / \mathrm{kg}$. ${ }^{13}$

The scientific data from this animal research in addition to the extensive anecdotal experience of successive generations of traditional Egyptian herbalists, prompted our research group, which has a special interest in traditional herbal medicines, to undertake a pilot clinical study to evaluate the sedative and hypnotic effects of $L$. sativa seed oil in patients complaining of sleeping difficulty with or without anxiety.

\section{Methods}

This was a prospective single-blind, randomized, placebo-controlled trial performed in two outpatient clinics in Alexandria, one at the Mamorah Psychiatric Hospital and the other at the Green Clinical Research Center. Sixty consecutive patients presenting with insomnia or sleeping difficulty were recruited for the study after careful medical history-taking and a physical examination. Patients were included if they were aged over 18 years and had a presenting complaint of insomnia, defined as frequently upset sleep quantity/quality or difficulty in initiating or sustaining a normal deep sleep pattern. Dementia of the Alzheimer's type was not an exclusion criterion if the patient had a supportive partner/caregiver. Patients with concomitant organic or neuropsychiatric disease necessitating use of tranquilizers, sedatives, anticonvulsants, or psychotropic agents were excluded. Patients who were concomitantly receiving any other conventional or alternative medical intervention which might influence the study outcome, including all other herbal products, hypnotherapy, acupuncture, homeopathy, and yoga, were also excluded. Patients were asked not to use any of these concomitant interventions during the course of the study, and were excluded from the analysis if they did so. Critically ill patients with severe heart, renal, or liver dysfunction were excluded, as were patients whose condition precluded verbal communication, unless they were accompanied by a supportive partner/caregiver. The protocol was approved by the local research ethical committee (IORG0006902 Green Clinic and Research Center IRB \#1), and each subject signed a consent form.

The patients were randomized into two treatment groups. Group $1(\mathrm{n}=30)$ received one Sedan capsule every night for 1 week and Group $2(\mathrm{n}=30)$ received a matching placebo. Randomization was done using a software-generated block randomization technique and the patients were blinded to their allocated drug. Double-blinding was not possible in this pilot study because the baseline examination, supply of medication, and final assessment were all carried out by the same investigator at each clinic.

\section{Outcome measures}

The effect of Sedan vs placebo on both the modified StateTrait Anxiety Inventory (STAI) score ${ }^{14}$ and a sleeping difficulty score was assessed at the end of 1 week of treatment. The modified STAI scale comprises 20 questions, each of which is answered on a five-point scale, from 1 (nil) to 4 (very much). The sleeping difficulty scale was a 10-item questionnaire based on the items of The Leeds Sleep Evaluation Questionnaire ${ }^{15}$ and assessed subjective patient estimates of and satisfaction with sleep quality using a three-point rating scale $(0=$ rare/nil, $1=$ sometimes, and $2=$ often $)$. The higher the total score on both scales, the worse the patient's insomnia was deemed to be. Both questionnaires were administered verbally at baseline and at the end of 1 week of treatment. Both instruments were translated into the Arabic language by the investigators and had been validated previously in patients with anxiety and insomnia treated at Mamorah Psychiatric Hospital (unpublished data). Clinical Global Impression of Change (CGI-C) scores were also recorded by the investigator at baseline after 1 week of treatment. The CGI-C is a sevenitem symptom rating scale $(0=$ not assessed, $1=$ very much 
improved, $2=$ much improved, $3=$ minimally improved, $4=$ no change, $5=$ minimally worse, $6=$ much worse, $7=$ very much worse).

\section{Statistical analysis}

Data were analyzed using the computer software package SPSS (v 14; SPSS, Inc, Chicago, IL). Sample size was calculated using SigmaStat $^{\circledR}$ (v 3.5 for Windows; Systat Software, Inc, Chicago, IL). The minimum sample size needed for each treatment group was calculated to be 23 subjects to detect a difference of 3.0 points in mean sleep scale scores with a standard deviation (SD) of 3.5, 80\% power, and a 0.05 two-tailed level of significance. A paired-samples $t$-test was done to compare the mean scores for each group before and after treatment. An independent-samples $t$-test was done to test for a significant difference between any improvements in mean score in each treatment group.

Multivariate analysis of covariance with treatment as the main independent factor and the study center as a controlling factor was used in full factorial model testing for main effects plus any possible interaction effects. Baseline pretreatment scores were used as covariates to control for initial group differences potentially affecting the dependent outcome variables, ie, any differences between baseline and posttreatment STAI and sleep scores, and their interaction. Parametric statistical methods were utilized after ascertaining the normality of distribution of the dependent variables by Kolmogorov-Smirnov goodness of fit test.

\section{Results}

Two patients from Group 1 and one from Group 2 withdrew from the study after randomization, and three patients in each group were lost to follow-up. One patient from Group 2 was not included in the statistical analysis as a result of recording errors made on the sleep scoring sheet. Twenty-five patients from each group were finally assessed, and their results were subjected to statistical analysis. The two groups were well matched for age, gender, and baseline sleep scores (see Table 1).

Table I Baseline characteristics of the study population

\begin{tabular}{llll}
\hline Characteristic & $\begin{array}{l}\text { L. sativa } \\
(\mathbf{n}=\mathbf{2 5})\end{array}$ & $\begin{array}{l}\text { Placebo } \\
(\mathbf{n}=\mathbf{2 5})\end{array}$ & P-value \\
\hline Age, years (mean \pm SD) & $62.5 \pm 8.5$ & $63.6 \pm 7.8$ & 0.636 \\
Gender (M/F) & $18 / 7$ & $17 / 8$ & 0.5 \\
Mean total STAI score & $58 \pm 8.6$ & $57 \pm 9.2$ & 0.693 \\
Mean total sleeping score & $11.84 \pm 6.2$ & $12.08 \pm 5.8$ & 0.888 \\
\hline
\end{tabular}

Abbreviations: M, male; F, female; STAI, State-Trait Anxiety Inventory; SD, standard deviation.
Cronbach's alpha coefficients for the 20 items on the modified STAI scale and for the ten items of the sleeping difficulty scale were 0.835 and 0.764 , respectively, indicating good reliability. There was a statistically significant improvement in mean scores for both the modified STAI and the sleeping difficulty questionnaires in both groups after treatment (Tables 2 and 3). However, improvements in both the mean modified STAI score and mean sleep score were significantly greater in Group 1 than in Group 2 by independent-samples $t$-test $(P<0.05)$.

Multivariate analysis was undertaken using treatment allocation as the main independent factor and the study center as a controlling factor in a full factorial model, with baseline scores as covariates (Tables 4 and 5). Treatment allocation and baseline scores (covariates) had statistically significant effects on the outcome variables, while both the study center and its two-way interaction with treatment (group, center) did not. Univariate effects of the corrected model and the treatment groups adjusted for baseline severity on both the STAI and sleep score showed statistically significant differences, while the study center and its twoway interaction with the groups did not.

Eighteen out of the 25 patients who completed the protocol (72\%) in Group 1 rated their insomnia as "very much or much improved" on the CGIC scale, versus $5(20 \%)$ in the control group (ie, $60 \%$ vs $16.7 \%$ respectively of the 30 intention-to-treat patients) (risk ratio: $3.6 ; 95 \%$ confidence interval: $1.58-8.18$ ).

\section{Discussion}

To our knowledge, this is the first published study of the effect of L. sativa seed oil on a sleeping disorder. We found that L. sativa seed oil significantly improved both STAI and sleep scores, with no apparent side effects at the dose strength used.

Being clinicians with a special interest in complementary alternative medicine, we undertook this pilot research on a pragmatic hypothesis-generating basis, rather than as a confirmatory or explanatory study, and hope that our findings will help pave the way for other investigators to join in the attempt to bridge the gap between alternative medicine and evidence-based medicine. This study has a number of limitations, including a small size, a short follow-up duration, and a single-blind design. The obvious reason for this is that, unlike patented new chemical entities with research funding from the pharmaceutical industry, quality research involving alternative medicines tends to suffer from financial and logistic constraints. 
Table 2 Paired-samples descriptive statistics for STAl and sleep scores

\begin{tabular}{|c|c|c|c|c|c|}
\hline Treatment group & & Paired variables & Mean & $\mathbf{n}$ & SD \\
\hline \multirow[t]{4}{*}{ Experimental } & Pair I & Total STAI score after treatment & 45.52 & 25 & 12.65 \\
\hline & & Total STAI score before treatment & 58.08 & 25 & 10.64 \\
\hline & Pair 2 & Total sleep score after treatment & 7.4 & 25 & 3.56 \\
\hline & & Total sleep score before treatment & 11.84 & 25 & 4.04 \\
\hline \multirow[t]{4}{*}{ Placebo } & Pair I & Total STAI score after treatment & 51.52 & 25 & 10.77 \\
\hline & & Total STAI score before treatment & 57 & 25 & 9.33 \\
\hline & Pair 2 & Total sleep score after treatment & 10.16 & 25 & 3.51 \\
\hline & & Total sleep score before treatment & 12.08 & 25 & 3.58 \\
\hline
\end{tabular}

Abbreviations: STAI, State-Trait Anxiety Inventory; SD, standard deviation.

Table 3 Paired-samples t-test for STAI and sleep scores

\begin{tabular}{|c|c|c|c|c|c|c|c|c|}
\hline \multirow[t]{2}{*}{ Treatment group } & \multirow{2}{*}{$\begin{array}{l}\text { Variable } \\
\text { pairs }\end{array}$} & \multicolumn{2}{|c|}{ Paired differences } & \multicolumn{2}{|l|}{$95 \% \mathrm{Cl}$} & \multirow[t]{2}{*}{$\mathbf{t}$} & \multirow[t]{2}{*}{ df } & \multirow[t]{2}{*}{$P$-value } \\
\hline & & Mean & SD & Lower & Upper & & & \\
\hline \multirow[t]{2}{*}{ Experimental } & Pair I & -12.56 & 12.23 & $-|7.6|$ & -7.51 & -5.13 & 24 & 0.00 \\
\hline & Pair 2 & -4.44 & 4.56 & -6.32 & -2.56 & -4.87 & 24 & 0.00 \\
\hline \multirow[t]{2}{*}{ Placebo } & Pair I & -5.48 & $|0.3|$ & -9.74 & -1.22 & -2.66 & 24 & 0.01 \\
\hline & Pair 2 & -1.92 & 4.48 & -3.77 & -0.07 & -2.14 & 24 & 0.04 \\
\hline
\end{tabular}

Abbreviations: SD, standard deviation; Cl, confidence interval; STAI, State-Trait Anxiety Inventory.

Table 4 Multivariate effects on outcome variables ${ }^{a}$

\begin{tabular}{llllll}
\hline Effect & & Value & F $^{\mathrm{b}}$ & Significance & Partial eta squared \\
\hline Baseline STAI score (covariate) & Pillai trace & 0.138 & 3.435 & 0.041 & 0.138 \\
& Hotelling trace & 0.16 & 3.435 & 0.041 & 0.138 \\
Baseline sleep score (covariate) & Pillai trace & 0.425 & 15.903 & 0.000 & 0.425 \\
& Hotelling trace & 0.74 & 15.903 & 0.000 & 0.425 \\
Treatment group (independent) & Pillai trace & 0.163 & 4.199 & 0.022 & 0.163 \\
& Hotelling trace & 0.195 & 4.199 & 0.022 & 0.163 \\
Study center & Pillai trace & 0.013 & 0.292 & 0.748 & 0.013 \\
Group*center & Hotelling trace & 0.014 & 0.292 & 0.748 & 0.013 \\
& Pillai trace & 0.015 & 0.318 & 0.729 & 0.015 \\
\hline
\end{tabular}

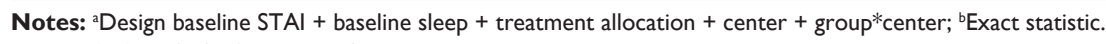

Abbreviation: STAI, State-Trait Anxiety Inventory.

Table 5 Univariate tests of between-subject effects

\begin{tabular}{|c|c|c|c|c|c|c|c|}
\hline Source & Dependent variable & $\begin{array}{l}\text { Type III sum } \\
\text { of squares }\end{array}$ & df & Mean square & $\mathbf{F}$ & $P$-value & $\begin{array}{l}\text { Partial eta } \\
\text { squared }\end{array}$ \\
\hline \multirow[t]{2}{*}{ Model } & $\begin{array}{l}\text { Effect on STAI score } \\
\text { after treatment }\end{array}$ & $6053.78^{a}$ & 6 & 1008.963 & 9.281 & 0.000 & 0.559 \\
\hline & $\begin{array}{l}\text { Effect on sleep score } \\
\text { after treatment }\end{array}$ & $1012.105^{\mathrm{b}}$ & 6 & 168.684 & 13.424 & 0.000 & 0.647 \\
\hline Baseline STAI score (covariate) & Effect on STAI score & 440.8265 & 1 & 440.827 & 4.055 & 0.050 & 0.084 \\
\hline Baseline sleep score (covariate) & Effect on sleep score & 380.3215 & 1 & 380.321 & 30.266 & 0.000 & 0.408 \\
\hline \multirow[t]{2}{*}{ Treatment group } & Effect on STAI score & 593.8728 & 1 & 593.873 & 5.463 & 0.024 & 0.110 \\
\hline & Effect on sleep score & 94.32857 & 1 & 94.329 & 7.507 & 0.009 & 0.146 \\
\hline \multirow[t]{2}{*}{ Study center } & Effect on STAI score & 60.64028 & 1 & 60.640 & 0.558 & 0.459 & 0.013 \\
\hline & Effect on sleep score & 0.658766 & 1 & 0.659 & 0.052 & 0.820 & 0.001 \\
\hline \multirow[t]{2}{*}{ Group*Center } & Effect on STAI score & 6.996249 & 1 & 6.996 & 0.064 & 0.801 & 0.001 \\
\hline & Effect on sleep score & 3.32109 & 1 & 3.321 & 0.264 & 0.610 & 0.006 \\
\hline \multirow[t]{2}{*}{ Error } & Effect on STAI score & 4783.22 & 44 & 108.710 & & & \\
\hline & Effect on sleep score & 552.8946 & 44 & 12.566 & & & \\
\hline \multirow[t]{2}{*}{ Total } & Effect on STAI score & 10837 & 50 & & & & \\
\hline & Effect on sleep score & 1565 & 50 & & & & \\
\hline
\end{tabular}

Notes: ${ }^{\mathrm{a}}{ }^{2} 0.559$ (adjusted 0.498); ${ }^{\mathrm{b}} \mathrm{R}^{2} 0.647$ (adjusted 0.599).

Abbreviation: STAI, State-Trait Anxiety Inventory. 
In our opinion, alternative medicines, in particular products already available as over-the-counter dietary supplements, should be subjected to more translational research and clinical trials in human subjects, and the results made available in the scientific literature. The criteria for publishing such studies would need to be different to those used with the registration trials for new chemical entities.

\section{Conclusion}

In conclusion, $L$. sativa seed oil was found to be a useful sleeping aid and may be a hazard-free line of treatment, especially in geriatric patients suffering mild-to-moderate forms of anxiety and sleeping difficulties. Larger randomized controlled studies are needed to confirm this.

\section{Disclosure}

Pharco Pharmaceutical Company, Alexandria, Egypt, supported this study by supplying the trial capsules free of charge.

\section{References}

1. Ancoli-Israel S, Roth T. Characteristics of insomnia in the United States: Results of the National Sleep Foundation Survey I. Sleep. 1999;22 (Supp1 2):S347-S353.

2. American Psychiatric Association. Diagnostic and Statistical Manual Of Mental Disorders. 4th ed. Washington, DC: American Psychiatric Association; 1994.
3. Vitiello MV. Sleep disorders and aging: Understanding the causes. J Gerontol A Biol Sci Med Sci. 1997;52A:M189-M191.

4. Foley DJ, Monjan AA, Brown SL, et al. Sleep complaints among elderly persons: An epidemiologic study of three communities. Sleep. 1995; 18:425-432.

5. Morgan K, Healey DW, Healey PJ. Factors influencing persistent subjective insomnia in old age: A follow up study of good and poor sleepers aged 65 to 74. Age Ageing. 1989;2:117-122.

6. Wingard DL, Berkman PT. Mortality risk associated with sleeping patterns among adults. Sleep. 1983;6:102-107.

7. Ramakrishnan K, Scheid DC. Treatment options for insomnia. Am Fam Physician. 2007;76:517-526.

8. Chittendon F. RHS Dictionary of Plants plus Supplement 1956. Oxford, UK: Oxford University Press; 1951.

9. Chiej R. Encyclopaedia of Medicinal Plants. Vancouver, CA: MacDonald Publishing; 1984.

10. Bown D. Encyclopaedia of Herbs and Their Uses. London, UK: Dorling Kindersley; 1995.

11. Grieve M. A Modern Herbal. London, UK: Jonathan Cape; 1931.

12. Sayyah M, Hadidi N, Kamalinejad M. Analgesic and anti-inflammatory activity of Lactuca sativa seed extract in rats. J Ethnopharmacol. 2004; 92:325-329.

13. Said SA, Kashef HE, Mazar ME, Salama O. Phytochemical and pharmacological studies on Lactuca sativa seed oil. Fitoterapia. 1996; 67:215-219.

14. Spielberger CD, Gorsuch RL, Lushene TE. State-Trait Anxiety Inventory. Madrid, Spain: TEA Ediciones; 1994.

15. Parrott AC, Hindmarch I. Factor analysis of a sleep evaluation questionnaire. Psychol Med. 1978;8:325-329. 


\section{Appendix \\ Sleeping scale questionnaire Describe your state during the last week, choosing 0 (rare/nil), I (sometimes), or 2 (often/always)}

1. Did you have difficulty in falling asleep after lying in bed?

2. Did you awaken frequently after falling asleep?

3. Did you awaken more than once during one night?

4. Did you awaken earlier than you should have?

5. Did you feel sleepy during the working hours?
6. Did you experience difficulty in concentration during the working hours?

7. Did you use or feel badly in need of additional sleeping aids?

8. Did you feel clumsy or drowsy after wakening in the morning?

9. Did you feel in need of frequent naps or a lie-down during the day?

10. Did you experience comfortable, quiet, and continuous sleep?*

*Reversed score

\section{Publish your work in this journal}

The International Journal of General Medicine is an international, peer-reviewed open-access journal that focuses on general and internal medicine, pathogenesis, epidemiology, diagnosis, monitoring and treatment protocols. The journal is characterized by the rapid reporting of reviews, original research and clinical studies across all disease areas.
A key focus is the elucidation of disease processes and management protocols resulting in improved outcomes for the patient.The manuscript management system is completely online and includes a very quick and fair peer-review system. Visit http://www.dovepress.com/ testimonials.php to read real quotes from published authors. 